\title{
Hyperbolicity of Physical Theories with Application to General Relativity
}

\author{
David Hilditch ${ }^{1}$ and Ronny Richter ${ }^{2}$ \\ ${ }^{1}$ Theoretical Physics Institute, University of Jena, 07743 Jena, Germany \\ ${ }^{2}$ Mathematisches Institut, Universiät Tübingen, 72076 Tübingen, Germany
}

\begin{abstract}
We consider gauge theories from the free evolution point of view, in which initial data satisfying constraints of a theory are given. Because the constraints are compatible with the field equations they remain so. We study a model constrained Hamiltonian theory and identify a particular structure in the equations of motion which we call the standard gauge freedom. The pure gauge subsystem of this model theory is identified and the manner in which the gauge variables couple to the field equations is presented. We demonstrate that the set of gauge choices that can be coupled to the field equations to obtain a, properly defined, wave-like formulation is exactly the set of wave-like pure gauges. Consequently we analyze a parametrized family of formulations of general relativity. The generalization of the harmonic gauge formulation to a five parameter family of gauge conditions is obtained.
\end{abstract}

PACS numbers: 95.30. Sf, 04.25.D-

I. Introduction. Field theories often have wave-like, or hyperbolic, degrees of freedom contained somehow in a set of variables, some of which are constrained, and some of which, the gauge fields, are not determined by the theory 1]. Physical states are equivalence classes of solutions related by a change of gauge. Solutions to the theory can be understood through properties of the equations of motion, which consist of a mixture of the gauge, constraint and physical quantities. Unraveling this structure in general may be hopeless. But if the gauge is carefully chosen, say by taking the harmonic gauge in general relativity [2], then the full set of equations of motion may be rendered strongly hyperbolic [3, 4]. This condition guarantees the existence of a unique solution to the initial value problem that depends continuously on the initial data, at least locally in time. As highlighted in 5] "Ideally, one would like to exhibit a kind of hyperbolic skeleton of the Einstein equations and a complete characterization of the freedom to fix the gauge from which all hyperbolic reductions should be derivable. Instead, there are at present various different methods available which have been invented to serve specific needs," this ad-hoc characterization is unsatisfactory. Equations of motion for the gauge choice can be obtained in the absence of any coupling to the theory, which begs the question what is this skeleton? In other words, what are the set of pure gauges that can be coupled to the theory to form a hyperbolic formulation? Since the basic characterization of a set of partial differential equations can be made in the linear approximation, we may start by directing our efforts there. We thus begin to address these issues in section II for a model linear constrained Hamiltonian system. In section III we examine conditions under which a formulation of the Hamiltonian theory is strongly hyperbolic. In section IV we apply our findings to general relativity (GR) with a five parameter family of gauge conditions and obtain the generalization of the harmonic formulation to this family.
II. A model theory with gauge freedom. Consider the equations of motion for the Hamiltonian density,

$$
\begin{aligned}
H= & \frac{1}{2}\left(\begin{array}{c}
\partial_{i} q \\
p
\end{array}\right)^{\dagger}\left(\begin{array}{cc}
V^{i j} & F^{\dagger i} \\
F^{j} & M^{-1}
\end{array}\right)\left(\begin{array}{c}
\partial_{j} q \\
p
\end{array}\right) \\
& +g_{q}^{\dagger} C_{\mathcal{H}} V^{i j} \partial_{i} \partial_{j} q+g_{p}^{\dagger} C_{\mathcal{M}}{ }^{i} M^{-1} \partial_{i} p
\end{aligned}
$$

with canonical positions and momenta $(q, p)$. Every matrix is constant, $M^{-1}$ is invertible and $F^{i}=\beta^{i} I$ for some shift vector $\beta^{i}$, with $I$ the appropriate identity. Such a Hamiltonian can be obtained from that of GR by linearizing [6] and discarding lower derivatives. Variation with respect to the gauge fields $\left(g_{q}, g_{p}\right)$ reveals the constraints

$$
\mathcal{H}=C_{\mathcal{H}} V^{i j} \partial_{i} \partial_{j} q=0, \quad \mathcal{M}=C_{\mathcal{M}}{ }^{i} M^{-1} \partial_{i} p=0,
$$

which we will take to be first class and call the Hamiltonian and momentum constraints respectively.

Gauge invariance: The constraints generate the gauge transformation,

$$
\begin{aligned}
& q \rightarrow \bar{q}=q-M^{-1} C_{\mathcal{M}}{ }^{\dagger i} \partial_{i} \psi, \\
& p \rightarrow \bar{p}=p-V^{i j} C_{\mathcal{H}}^{\dagger} \partial_{i} \partial_{j} \theta,
\end{aligned}
$$

with unspecified fields $\theta$ and $\psi$. We require that the field strength $V^{i j} \partial_{i} \partial_{j} q$ and curl $\epsilon^{i} M^{-1} \partial_{i} p$, defined by some square anti-hermitian matrices $\epsilon^{i}$, are invariant under this transformation. Gauge invariance thus gives,

$$
\begin{aligned}
& \left(A_{\mathcal{H} \mathcal{M}}\right)^{(i} C_{\mathcal{M}}{ }^{j)}=C_{\mathcal{H}} V^{i j}, \quad V^{(i j} M^{-1} C_{\mathcal{M}}{ }^{\dagger k)}=0, \\
& \epsilon^{(i} M^{-1} V^{j k)} C_{\mathcal{H}}^{\dagger}=0,
\end{aligned}
$$

for some matrices $\left(A_{\mathcal{H M}}\right)^{i}$, where the index parentheses denote symmetrization. Gauge invariance of the evolution equations also implies that

$$
\begin{aligned}
\partial_{t} \theta & =\beta^{i} \partial_{i} \theta+\bar{g}_{q}, \\
\partial_{t} \psi & =\left(A_{\mathcal{H} \mathcal{M}}\right)^{\dagger i} \partial_{i} \theta+\beta^{i} \partial_{i} \psi+\bar{g}_{p},
\end{aligned}
$$

where $\bar{g}_{q}, \bar{g}_{p}$ denote the change under (2), and where here, and in what follows, $\beta^{i}$ is taken to include the appropriate identity. 
Electric and magnetic degrees of freedom: Without loss of generality the matrix $C_{\mathcal{H}}$ has linearly independent rows, so we can decompose the potential matrix $V^{i j}$ according to

$$
V^{i j}=V_{\mathcal{P}}^{i j}+\hat{C}_{\mathcal{H}}^{\dagger} \hat{C}_{\mathcal{H}} V^{i j}, \quad V_{\mathcal{P}}^{i j}=\perp_{\mathcal{P}} V^{i j},
$$

with $\hat{C}_{\mathcal{H}}=\left[C_{\mathcal{H}} C_{\mathcal{H}}^{\dagger}\right]^{-1 / 2} C_{\mathcal{H}}$, and the projection operator $\perp_{\mathcal{P}}$ defined implicitly by (4). In the absence of Hamiltonian constraints we assume that $V^{i j}=V_{\mathcal{P}}^{i j}$, and always that $V_{\mathcal{P}}^{i j}=\epsilon^{\dagger i}\left(A_{V B}\right) \epsilon^{j}+C_{\mathcal{M}}^{\dagger(i}\left(A_{V \mathcal{M}}\right) C_{\mathcal{M}}{ }^{j)}$ for some Hermitian matrices $\left(A_{V B}\right)$ and $\left(A_{V \mathcal{M}}\right)$. The electric and magnetic degrees of freedom are,

$$
E=V_{\mathcal{P}}^{i j} \partial_{i} \partial_{j} q, \quad B=\epsilon^{i} M^{-1} \partial_{i} p .
$$

which, up to coupling to the constraints, form a closed subsystem and are gauge invariant. Such fields can be similarly defined in the absence of Hamiltonian constraints. They are not used in the analysis that follows.

Closure of the pure gauge subsystem: We call an equation of motion for the gauge fields a gauge choice. Here we consider only evolution conditions

$$
\begin{aligned}
& \partial_{t} g_{q}=\left(A_{g_{q} g_{q}}\right)^{i} \partial_{i} g_{q}+\left(A_{g_{q} g_{p}}\right)^{i} \partial_{i} g_{p}+\left(A_{g_{q} p}\right) p, \\
& \partial_{t} g_{p}=\left(A_{g_{p} g_{q}}\right)^{i} \partial_{i} g_{q}+\left(A_{g_{p} g_{p}}\right)^{i} \partial_{i} g_{p}+\left(A_{g_{p} q}\right)^{i} \partial_{i} q .
\end{aligned}
$$

We assume that $\left(A_{g_{q} p}\right)=A C_{\mathcal{H}}$ and $\left(A_{g_{p} q}\right)^{i}=B C_{\mathcal{M}^{i}}+$ $C^{i} C_{\mathcal{H}} M$ for some matrices $A, B$ and $C^{i}$, a restriction which can be dropped by altering our arguments slightly. Assume that we are given a solution to the theory. We have already seen that the field equations are invariant under the gauge transformation (2). The pure gauge subsystem (3) is closed by substituting the gauge difference from (2) into (5), taking $g_{q} \rightarrow \bar{g}_{q}$ and $g_{p} \rightarrow \bar{g}_{p}$.

Free evolution on the expanded phase space: We are free to modify the dynamics of the model theory away from the constraint satisfying hypersurface in phase space, provided that the constraint subsystem remains closed. We define new constraints $(\Theta, Z)$ with the same length as $\left(g_{q}, g_{p}\right)$ respectively. We couple the new constraints to the gauge conditions (3) by parametrized addition according to

$$
\begin{aligned}
\partial_{t} g_{q}= & \left(A_{g_{q} g_{q}}\right)^{i} \partial_{i} g_{q}+\left(A_{g_{q} g_{p}}\right)^{i} \partial_{i} g_{p}+\left(A_{g_{q} p}\right) p \\
& +\left(A_{g_{q} \Theta} \Theta \Theta\right. \\
\partial_{t} g_{p}= & \left(A_{g_{p} g_{q}}\right)^{i} \partial_{i} g_{q}+\left(A_{g_{p} g_{p}}\right)^{i} \partial_{i} g_{p}+\left(A_{g_{p} q}\right)^{i} \partial_{i} q \\
& +\left(A_{g_{p}} Z\right) Z .
\end{aligned}
$$

Likewise for the equations of motion

$$
\begin{aligned}
\partial_{t} q= & M^{-1} p+F^{i} \partial_{i} q-M^{-1} C_{\mathcal{M}}^{\dagger i} \partial_{i} g_{p}+\left(A_{q \Theta}\right) \Theta \\
\partial_{t} p= & V^{i j} \partial_{i} \partial_{j} q+F^{i} \partial_{i} p-V^{i j} C_{\mathcal{H}}^{\dagger} \partial_{i} \partial_{j} g_{q}+\left(A_{p Z}\right)^{i} \partial_{i} Z \\
& +\left(A_{p \mathcal{H}}\right) \mathcal{H} .
\end{aligned}
$$

We choose equations of motion for the new constraints

$$
\begin{aligned}
& \partial_{t} \Theta=\beta^{i} \partial_{i} \Theta+\left(A_{\Theta Z}\right)^{i} \partial_{i} Z+\left(A_{\Theta \mathcal{H}}\right) \mathcal{H}, \\
& \partial_{t} Z=\left(A_{Z \Theta}\right)^{i} \partial_{i} \Theta+\beta^{i} \partial_{i} Z+\left(A_{Z \mathcal{M}}\right) \mathcal{M} .
\end{aligned}
$$

The constraint subsystem is closed by

$$
\begin{aligned}
\partial_{t} \mathcal{H} & =\left(A_{\mathcal{H} \Theta}\right)^{i j} \partial_{i} \partial_{j} \Theta+\beta^{i} \partial_{i} \mathcal{H}+\left(A_{\mathcal{H} \mathcal{M}}\right)^{i} \partial_{i} \mathcal{M}, \\
\partial_{t} \mathcal{M} & =\left(A_{\mathcal{M} Z}\right)^{i j} \partial_{i} \partial_{j} Z+\left(A_{\mathcal{M H}}\right)^{i} \partial_{i} \mathcal{H}+\beta^{i} \partial_{i} \mathcal{M},
\end{aligned}
$$

with matrices

$$
\begin{aligned}
\left(A_{\mathcal{H} \Theta}\right)^{i j} & =C_{\mathcal{H}} V^{i j}\left(A_{q \Theta}\right), \\
\left(A_{\mathcal{M Z}}\right)^{i j} & =C_{\mathcal{M}}{ }^{(i} M^{-1}\left(A_{p Z}\right)^{j)}, \\
\left(A_{\mathcal{M H}}\right)^{i} & =C_{\mathcal{M}}{ }^{i} M^{-1}\left(A_{p \mathcal{H}}\right) .
\end{aligned}
$$

Natural choice of variables: The next assumption is that the variables can be appropriately broken up. For this we assume that for every unit spatial vector $s^{i}$, the rows of $C_{\mathcal{H}}$ and $C_{\mathcal{M}}{ }^{s} \equiv C_{\mathcal{M}}{ }^{i} s_{i}$ are contained in the span of the union of the rows of $V=$ $C_{\mathcal{H}} V^{s s}$ and $W=C_{\mathcal{M}}{ }^{s} M^{-1}$, which each have themselves independent rows, and furthermore that the contractions $X=V C_{\mathcal{H}}^{\dagger}$ and $Y=W C_{\mathcal{M}^{\dagger s}}$ are invertible. With these conditions we can define

$$
\begin{aligned}
C_{\theta} & =-X^{-1} C_{\mathcal{H}}, \\
C_{\psi} & =-Y^{-1} C_{\mathcal{M}}{ }^{s}+\left(A_{\mathcal{H} M}\right)^{\dagger s} C_{\theta}\left[M-C_{\mathcal{M}}{ }^{\dagger s} Y^{-1} C_{\mathcal{M}}{ }^{s}\right], \\
\perp & =I-V^{\dagger}\left[V V^{\dagger}\right]^{-1} V-W^{\dagger}\left[W W^{\dagger}\right]^{-1} W,
\end{aligned}
$$

and the decomposition of $\partial_{s} q$ and $p$ into gauge, constraint, and physical degrees of freedom,

$$
\begin{aligned}
\partial_{s}^{2} \theta & =C_{\theta} p+\left(A_{\theta \Theta}\right) \Theta, & \partial_{s}^{2} \psi & =C_{\psi} \partial_{s} q+\left(A_{\psi Z}\right) Z, \\
\mathcal{H} & =V \partial_{s} q, & \mathcal{M} & =W p, \\
\partial_{s} P_{q} & =\perp \partial_{s} q, & P_{p} & =\perp p,
\end{aligned}
$$

is invertible. The names here serve only to identify the relationship between the pure gauge and constraints.

Principal symbol of a formulation: Once the gauge and constraint addition parameters are fixed we say that we have a formulation of the theory. The principal symbol of a formulation in the $s^{i}$ direction is

$$
P^{s}=\left(\begin{array}{ccc}
P_{\mathcal{G}}^{s} & P_{\mathcal{G} \mathcal{C}}^{s} & 0 \\
0 & P_{\mathcal{C}}^{s} & 0 \\
0 & 0 & P_{\mathcal{P}}^{s}
\end{array}\right)
$$

We assume that the constraint addition parameters are annihilated by the projection operator $\perp$. This restriction can also be relaxed. The pure gauge sub-block,

$$
P_{\mathcal{G}}^{s}=\left(\begin{array}{cccc}
\beta^{s} & 0 & I & 0 \\
\left(A_{\mathcal{H M}}\right)^{\dagger s} & \beta^{s} & 0 & I \\
-\left(A_{g_{q} p}\right) V^{\dagger} & 0 & \left(A_{g_{q} g_{q}}\right)^{s} & \left(A_{g_{q} g_{p}}\right)^{s} \\
0 & -\left(A_{g_{p} q}\right)^{s} W^{\dagger} & \left(A_{g_{p} g_{q}}\right)^{s} & \left(A_{g_{p} g_{p}}\right)^{s}
\end{array}\right)
$$


is exactly the principal symbol of the pure gauge subsystem described after equation (5). The off-diagonal block,

$$
P_{\mathcal{G C}}^{s}=\left(\begin{array}{cccc}
0 & \left(A_{\theta Z}\right) & \left(A_{\theta \mathcal{H}}\right) & 0 \\
\left(A_{\psi \Theta}\right) & 0 & 0 & \left(A_{\psi \mathcal{M}}\right) \\
\left(A_{\Theta}\right) & 0 & 0 & 0 \\
0 & \left(A_{Z}\right) & 0 & 0
\end{array}\right)
$$

with sub-matrices,

$$
\begin{aligned}
\left(A_{\theta Z}\right) & =\left(A_{\theta \Theta}\right)\left(A_{\Theta Z}\right)+C_{\theta}\left(A_{p Z}\right)^{s}, \\
\left(A_{\theta \mathcal{H}}\right) & =\left(A_{\theta \Theta}\right)\left(A_{\Theta \mathcal{H}}\right)-X^{-1}+C_{\theta}\left(A_{p \mathcal{H}}\right), \\
\left(A_{\psi \Theta}\right) & =\left(A_{\psi Z}\right)\left(A_{\psi \Theta}\right)-\left(A_{\mathcal{H} \mathcal{M}}\right)^{\dagger s}\left(A_{\theta \Theta}\right)+C_{\psi}\left(A_{g_{q} \Theta}\right), \\
\left(A_{\psi \mathcal{M}}\right) & =\left(A_{\psi Z}\right)\left(A_{Z \mathcal{M}}\right)-Y^{-1}-\left(A_{\mathcal{H M}}\right)^{\dagger s} C_{\theta} C_{\mathcal{M}}{ }^{\dagger s} Y^{-1}, \\
\left(A_{\Theta}\right) & =\left(A_{g_{q} p}\right) V^{\dagger}\left(A_{\theta \Theta}\right)+\left(A_{g_{q} \Theta}\right), \\
\left(A_{Z}\right) & =\left(A_{g_{p} q}\right)^{s} W^{\dagger}\left(A_{\psi Z}\right)+\left(A_{g_{p} Z}\right),
\end{aligned}
$$

parametrizes the coupling of the gauge fields to the constraints. The constraint violating sub-block,

$$
P_{\mathcal{C}}^{s}=\left(\begin{array}{cccc}
\beta^{s} & \left(A_{\Theta Z}\right)^{s} & \left(A_{\Theta \mathcal{H}}\right) & 0 \\
\left(A_{Z \Theta}\right)^{s} & \beta^{s} & 0 & \left(A_{Z \mathcal{M}}\right) \\
\left(A_{\mathcal{H} \Theta}\right)^{s s} & 0 & \beta^{s} & \left(A_{\mathcal{H} \mathcal{M}}\right)^{s} \\
0 & \left(A_{\mathcal{M} Z}\right)^{s s} & \left(A_{\mathcal{M H}}\right)^{s} & \beta^{s}
\end{array}\right)
$$

is exactly the principal symbol of the constraint subsystem. Finally the physical sub-block,

$$
P_{\mathcal{P}}^{s}=\left(\begin{array}{cc}
\beta^{s} & \perp M^{-1} \\
\perp V^{s s} & \beta^{s}
\end{array}\right),
$$

contains neither constraint addition or gauge parameters.

Strong hyperbolicity: A necessary condition for strongly hyperbolicity is that $P^{s}$ has real eigenvalues and a complete set of eigenvectors for every $s^{i}$. Strong hyperbolicity is equivalent to well-posedness, that is existence of a unique solution depending continuously on the given data, of the initial value problem [3, 4, 7].

III. Basic properties of theories with the standard gauge freedom. Consider the theory of the previous section. Then:

Lemma. No formulation is strongly hyperbolic if the physical sub-block is not.

Proof. Obviously a necessary condition for diagonalizability with real eigenvalues of (6), for any formulation, is that of $P_{\mathcal{P}}^{s}$.

Lemma. A necessary condition for strong hyperbolicity of a formulation is that the pure gauge and constraint violating subsystems are strongly hyperbolic.

Proof. We need to show that if the matrix (6) is diagonalizable with real eigenvalues then this property holds for the pure gauge and constraint violating sub-blocks. A diagonalizable upper block triangular matrix has diagonalizable blocks on the diagonal [4, App. A]. Moreover, the set of eigenvalues of the full matrix is the union of the eigenvalues of the diagonal blocks. The lemma follows.

Definition. If for every strongly hyperbolic pure gauge there exists a choice of constraint addition parameters so that the formulation is strongly hyperbolic, we say that the theory has the standard gauge freedom.

Theorem. Given a theory with the standard gauge freedom, a pure gauge can be used to form a strongly hyperbolic formulation if and only if it is strongly hyperbolic.

Proof. The result follows trivially from the Lemmas and the definition of the standard gauge freedom.

IV. Application to GR. The ADM Hamiltonian 8 for vacuum $\mathrm{GR}$ is $H_{\mathrm{ADM}}=-\alpha H+2 \beta^{i} M_{i}$, with Hamiltonian and momentum constraints

$$
H=R-K_{i j} K^{i j}+K^{2}, \quad M_{i}=D^{j} K_{i j}-D_{i} K .
$$

Gauge freedom in the non-linear regime: We take the freedom to be to choose coordinates $x^{\mu}=\left(t, x^{i}\right)$ on spacetime; qualitative features of the model carry over. The constraints are obviously spatially covariant. Given an additional upper case time coordinate $T$ with normal vector $N^{a}$ such that $N^{a}=W\left(n^{a}+v^{a}\right)$, with Lorentz factor $W$ and spatial boost vector $v^{i}$ then

$$
\begin{aligned}
{ }^{(N)} H & =W^{2} H-2 W^{2} M_{v}, \\
\perp \cdot{ }^{(N)} M_{i} & =W M_{i}+2 W^{3} M_{v} v_{i}-W^{3} H v_{i},
\end{aligned}
$$

where $\perp_{b}^{a}$ is the projection operator into slices of constant $t$, and subscript $v$ denotes contraction with the velocity $v^{i}$. The electric and magnetic parts of the Weyl tensor [9], form a closed subsystem, up to coupling to the constraints, and from the point of view of the lower case observer the spatial part of the upper case electric and magnetic parts are

$$
\begin{gathered}
\perp{ }^{(N)} E_{i j}=\left(2 W^{2}-1\right) E_{i j}-2 W^{2} E_{v(i} v_{j}+W^{2} E_{v v} \gamma_{i j} \\
\quad+2 W^{2} \epsilon^{k}{ }_{v i} B_{j) k}, \\
\perp{ }^{(N)} B_{i j}=W^{2} B_{i j}-W^{2} \epsilon^{k}{ }_{i j} E_{k v}-W^{2} \epsilon^{k}{ }_{v i} E_{j k},
\end{gathered}
$$

which shows that if the fields vanish in one foliation they vanish in every foliation.

Linearized pure gauge subsystem: The linearized pure gauge subsystem is [10],

$$
\begin{aligned}
\partial_{t} \theta & =U-\psi_{i} D^{i} \alpha+\beta^{i} \partial_{i} \theta, \\
\partial_{t} \psi^{i} & =V^{i}+\alpha D^{i} \theta-\theta D^{i} \alpha+\mathcal{L}_{\beta} \psi^{i},
\end{aligned}
$$

where $\theta=-n_{a} \Delta\left[x^{a}\right], \psi^{i}=-\perp_{a}^{i} \Delta\left[x^{a}\right], U=\Delta[\alpha]$ and $V^{i}=\Delta\left[\beta^{i}\right]$. Under an infinitesimal change of gauge the perturbation to the metric and extrinsic curvature are given by the York equations [11] with $\alpha \rightarrow \theta$ and $\beta^{i} \rightarrow$ $\psi^{i}$, which can be used to close the linearized pure gauge subsystem once we act on the gauge condition with the perturbation operator $\Delta$. 
Free evolution in the expanded phase space: We expand the phase space by constraints $\Theta$ and $Z_{i}$, and parametrize the equations of motion for the gauge by

$$
\begin{aligned}
\partial_{t} \alpha=- & g_{1} \alpha^{2} K+g_{2} \alpha \partial_{i} \beta^{i}+\beta^{i} \partial_{i} \alpha+2 c_{1} \alpha^{2} \Theta \\
\partial_{t} \beta^{i}= & \alpha^{2}\left[g_{3} \gamma^{k l} \gamma^{i j}+g_{4} \gamma^{i l} \gamma^{j k}\right] \partial_{l} \gamma_{j k}-g_{5} \alpha \partial^{i} \alpha+\beta^{j} \partial_{j} \beta^{i} \\
& +2 \alpha^{2} c_{2} Z^{i}
\end{aligned}
$$

with $g_{1}>0$ and $\bar{g}_{3}=2\left(g_{3}+g_{4}\right)>0$, and for the remaining variables by

$$
\begin{aligned}
\partial_{t} \gamma_{i j}= & -2 \alpha K_{i j}+\mathcal{L}_{\beta} \gamma_{i j}+\frac{1}{3} c_{3} \alpha \gamma_{i j} \Theta, \\
\partial_{t} K_{i j}=- & D_{i} D_{j} \alpha+\alpha\left[R_{i j}-2 K^{k}{ }_{i} K_{j k}+K_{i j} K\right]+\mathcal{L}_{\beta} K_{i j} \\
& \quad+2 c_{4} \alpha \partial_{(i} Z_{j)}+\frac{1}{3} c_{5} \alpha \gamma_{i j} \partial_{k} Z^{k}+\frac{1}{3} c_{6} \alpha \gamma_{i j} H, \\
\partial_{t} \Theta= & c_{7} \alpha H+c_{8} \alpha \partial_{i} Z^{i}+\mathcal{L}_{\beta} \Theta, \\
\partial_{t} Z_{i}= & c_{9} \alpha M_{i}+c_{10} \alpha \partial_{i} \Theta+\mathcal{L}_{\beta} Z_{i} .
\end{aligned}
$$

Strong hyperbolicity for non-linear and variable coefficient systems is defined, with additional smoothness conditions, by linearizing and working in the high-frequency frozen coefficient approximation [7]. In this approximation the Hamiltonian density [6] has the structure of (11).

Strong hyperbolicity of the pure gauge subsystem: The principal symbol of the linearized pure gauge subsystem (77) with gauge choice (8), where one must ignore constraint addition, has eigenvalues $\pm \sqrt{g_{3}}, \pm v_{ \pm}$, with

$$
\begin{aligned}
2 v_{ \pm}^{2}= & g_{1}+\bar{g}_{3}-g_{2} g_{5} \\
& \pm \sqrt{\left(g_{1}+\bar{g}_{3}-g_{2} g_{5}\right)^{2}-4\left(g_{1}-g_{2}\right) \bar{g}_{3}} .
\end{aligned}
$$

The subsystem is strongly hyperbolic if $g_{3}>0$ and either

$$
\begin{aligned}
& \text { i). } 0 \neq g_{2}<g_{1} \text { and } g_{2} g_{5}<g_{1}-2 \sqrt{g_{1}-g_{2}} \sqrt{\bar{g}_{3}}+\bar{g}_{3}, \\
& \text { ii). } g_{2}=0 \text { and } \bar{g}_{3} \neq g_{1} \text { or } g_{2}=0, \bar{g}_{3}=g_{1} \text { and } g_{5}=1 \text {. }
\end{aligned}
$$

the second clause of case ii). is that of generically distinct eigenvalues colliding without loss of diagonalizability.

Strong hyperbolicity of the constraint subsystem with vanishing gauge-constraint coupling: Choosing

$$
\begin{aligned}
& c_{1}=g_{1}, \quad c_{2}=g_{3}, \quad c_{3}=c_{5}=c_{6}=0 \\
& c_{4}=2 c_{7}=c_{8}=c_{9}=1, \quad c_{10}=2\left(1+\frac{g_{4}}{g_{3}}\right),
\end{aligned}
$$

guarantees both that the off-diagonal block of the principal symbol $P_{\mathcal{G C}}^{s}$ vanishes and that the constraint subsystem is strongly hyperbolic. The eigenvalues of the constraint violating sub-block $P_{\mathcal{C}}^{s}$ are $\pm \sqrt{c_{10}}$, which are guaranteed to be real inside the class of gauges we are considering, and \pm 1 with multiplicity three.

Strong hyperbolicity of physical sub-block: The physical sub-block is diagonalizable with eigenvalues \pm 1 , at least up to a trivial normalization. Assuming smoothness of the background implies the continuity requirement for strong hyperbolicity in every block.
Discussion: The choice (9) is the natural extension of the harmonic gauge formulation [2] to the family of gauge conditions (8). If a gauge in which the contracted Christoffel symbol is chosen to appear in the shift condition, i.e when $g_{4}=-\frac{1}{2} g_{3}$, the constraint addition parameters correspond to those of the principal part of the $\mathrm{Z} 4$ formulation [12]. Otherwise it differs in the constraint subsystem.

$\boldsymbol{V}$. Conclusion. Stimulated by [5], in which the possibility of identifying every hyperbolic formulation of GR was suggested, we identified a particular structure in constrained Hamiltonian equations of motion. We examined how pure gauge is inherited by a formulation of a theory. With this structure the set of strongly hyperbolic pure gauges are exactly those that can be used to form a strongly hyperbolic formulation, in-line with the expectation of the physicist. We expect that the results can be generalized to include elliptic gauges. It will furthermore be of interest to treat the initial boundary value problem. We used our findings to investigate hyperbolicity of a family of formulations of GR, generalizing [13] to non Hamiltonian formulations. Open questions include those relating to long-term existence with different gauges.

Acknowledgments. The authors thank S. Bernuzzi, B. Brügmann, C. Gundlach, N. Ó Murchada, M. Ruiz and A. Weyhausen for helpful discussions. The work was partially supported by DFG grant SFB/Transregio 7.

[1] R. P. Geroch, in General Relativity, edited by G. Hall (1996), gr-qc/9602055.

[2] Y. Bruhat, Acta Mathematica 88, 141 (1952).

[3] B. Gustafsson, H.-O. Kreiss, and J. Oliger, Time dependent problems and difference methods (Wiley, New York, 1995).

[4] C. Gundlach and J. M. Martín-García, Class. Quantum Grav. 23, S387 (2006), gr-qc/0506037.

[5] H. Friedrich and A. D. Rendall, Lect. Notes Phys. 540, 127 (2000), gr-qc/0002074.

[6] V. Moncrief, Ann. Phys. 88, 323 (1974).

[7] O. Sarbach and M. Tiglio, Living Reviews in Relativity 15 (2012), 1203.6443, URL http://www.livingreviews . org/lrr-2012-9

[8] R. Arnowitt, S. Deser, and C. W. Misner, in Gravitation: An Introduction to Current Research, edited by L. Witten (Wiley, New York, 1962), pp. 227-265.

[9] R. Maartens and B. A. Bassett, Class.Quant.Grav. 15, 705 (1998), gr-qc/9704059.

[10] A. M. Khokhlov and I. D. Novikov, Class. Quantum Grav. 19, 827 (2002).

[11] J. W. York, in Sources of gravitational radiation, edited by L. L. Smarr (Cambridge University Press, Cambridge, UK, 1979), pp. 83-126, ISBN 0-521-22778-X.

[12] C. Bona, T. Ledvinka, C. Palenzuela, and M. Žáček, Phys. Rev. D 67, 104005 (2003), gr-qc/0302083.

[13] D. Hilditch and R. Richter, Phys.Rev. D86, 123017 (2012), 1002.4119. 